\title{
Directed Percolation: Calculation of Feynman Diagrams in the Three-Loop Approximation
}

\author{
Loran Ts. Adzhemyan ${ }^{1}$, Michal Hnatičč,3,4, Mikhail V. Kompaniets ${ }^{1}$, Tomáš Lučivjanský ${ }^{4,5}$, and \\ Lukáš Mižišin ${ }^{2,3, \star}$ \\ ${ }^{1}$ Saint Petersburg State University, St. Petersburg, Russia \\ ${ }^{2}$ Bogoliubov Laboratory of Theoretical Physics, JINR, Dubna, Russia \\ ${ }^{3}$ Slovak academy of Sciences, Institute of Experimental Physics, Košice, Slovak Republic \\ ${ }^{4}$ Faculty of Sciences, P. J. Šafárik University in Košice, Košice, Slovak Republic \\ ${ }^{5}$ Peoples' Friendship University of Russia (RUDN University), Moscow, Russia
}

\begin{abstract}
The directed bond percolation process is an important model in statistical physics. By now its universal properties are known only up to the second-order of the perturbation theory. Here, our aim is to put forward a numerical technique with anomalous dimensions of directed percolation to higher orders of perturbation theory and is focused on the most complicated Feynman diagrams with problems in calculation. The anomalous dimensions are computed up to three-loop order in $\varepsilon=4-d$.
\end{abstract}

\section{Introduction}

The directed bond percolation process (DP) constitutes one of the simplest non-equilibrium systems in statistical physics [1-3]. It is one of the basic examples of an absorbing phase transition between an absorbing and active state. The DP universal class is important for the non-equilibrium physics and it may be considered as important as the Ising model for the static equilibrium systems.

An important method for an analysis of the second order phase transition is the renormalization group (RG) approach [4] and the $\varepsilon$-expansion where $\varepsilon$ is the deviation from the upper critical dimension $d_{c}=4$. In this work, the third-order pertubative theory is calculated numerically in the null-momentum (NM) subtraction scheme. By means of the scheme the renormalized Green function is obtained by $\mathcal{R}$ operation [4, 5] on Green function. Within this procedure anomalous dimensions $\gamma$ are expressed in terms of irreducible renormalized Feynman diagrams, and thus the calculation of renormalization constants can be entirely skipped $[6,7]$. The universal quantities are calculable in the form of the $\varepsilon$-expansion and they are independent of the choice of the renormalization scheme [4].

\section{Model and Method}

A field theoretical formulation of the percolation process [1-3] is based on the action functional,

$$
S_{0}=\tilde{\psi}\left(-\partial_{t}+D_{0} \partial^{2}-D_{0} \tau_{0}\right) \psi+\frac{D_{0} \lambda_{0}}{2}\left[\tilde{\psi}^{2} \psi-\tilde{\psi} \psi^{2}\right]
$$

\footnotetext{
${ }^{\star}$ e-mail: lukas.mizisin@gmail.com
} 
where $\partial^{2}$ is the Laplace operator, $\partial_{t}$ is differentiation with respect to time, $\psi>0$ is the coarse-grain density of percolating particles, $\tilde{\psi}$ is an auxiliary (Martin-Siggia-Rose) response field [9], $D_{0}$ is a diffusion coefficient, $\lambda_{0}$ is a positive coupling constant and $\tau_{0}$ is a deviation from the threshold value (an analog of critical temperature in static models [4]). The model is studied near its upper critical dimension $d_{c}=4$ using expansion in the formally small parameter $\varepsilon=d_{c}-d=4-d$.

The renormalized action functional for the DP process (1) can be written in the form

$$
S_{R}=\tilde{\psi}\left(-Z_{1} \partial_{t}+Z_{2} D \partial^{2}-Z_{3} D \tau\right) \psi+\frac{Z_{4} D \lambda \mu^{\varepsilon / 2}}{2}\left[\tilde{\psi}^{2} \psi-\tilde{\psi} \psi^{2}\right]
$$

On the other hand, the action functional $S_{R}$ can also be obtained by the standard procedure [4] of multiplicative renormalization of all fields and parameters

$$
\psi_{0}=\psi Z_{\psi}, \quad \tilde{\psi}_{0}=\tilde{\psi} Z_{\tilde{\psi}}, \quad D_{0}=D Z_{D}, \quad \lambda_{0}=\lambda \mu^{\varepsilon / 2} Z_{\lambda}, \quad \tau_{0}=\tau Z_{\tau}+\tau_{c},
$$

supplemented by similar relations between renormalization constants for terms in (2). From a direct comparison we can easily express RG constants for the model parameters in (3) through $Z_{i}, i=1, \ldots, 4$.

In this work, a renormalization scheme at the normalization point (NP), $p=0, \omega=0$ and $\tau=\mu^{2}$ is considered. The counterterms are then specified at the normalization point, and for numerical calculation it is advantageous to express the renormalization constants in terms of normalized Green functions

$$
\bar{\Gamma}_{1}=\left.\partial_{i \omega} \Gamma_{\tilde{\psi} \psi}\right|_{\omega=0} ^{p=0}, \quad \bar{\Gamma}_{2}=\left.\frac{-1}{2 D} \partial_{p}^{2} \Gamma_{\tilde{\psi} \psi}\right|_{\omega=0} ^{p=0}, \quad \bar{\Gamma}_{3}=\left.\frac{\left.\Gamma_{\tilde{\psi} \psi}\right|_{\tau=0}-\Gamma_{\tilde{\psi} \psi}}{D \tau}\right|_{\omega=0} ^{p=0}, \quad \bar{\Gamma}_{4}=\left.\frac{\Gamma_{\tilde{\psi} \tilde{\psi} \psi}-\Gamma_{\tilde{\psi} \psi \psi}}{D \lambda \mu^{\varepsilon / 2}}\right|_{\omega=0} ^{p=0},
$$

that satisfy the conditions $\left.\bar{\Gamma}_{i}^{R}\right|_{\tau=\mu^{2}}=1, i=1,2,3,4$. The renormalization constant is defined in this way and does not depend on $\tau$, as in the minimal subtraction (MS) scheme: The renormalization group equations are the same as in the MS scheme:

$$
\left(\mu \partial_{\mu}+\beta_{u} \partial_{u}-\tau \gamma_{\tau} \partial_{\tau}-D \gamma_{D} \partial_{D}\right) \Gamma_{i}^{R}=\left(n_{\psi} \gamma_{\psi}+n_{\tilde{\psi}} \gamma_{\tilde{\psi}}\right) \Gamma_{i}^{R},
$$

where $\mu$ is the renormalization mass, $n_{\psi}$ and $n_{\tilde{\psi}}$ are the numbers of the corresponding fields in Green function, $\gamma_{x}=\mu \partial_{\mu} \log Z_{x}$ is anomalous dimension and $\beta_{u}=\mu \partial_{\mu} u=u\left(-\epsilon-\gamma_{u}\right)$ is describing a flow of the charge $u=\lambda^{2}$. By applying these equations, one can obtain relations for the normalized functions

$$
\left(\mu \partial_{\mu}+\beta_{u} \partial_{u}-\tau \gamma_{\tau} \partial_{\tau}-D \gamma_{D} \partial_{D}\right) \bar{\Gamma}_{i}^{R}=\gamma_{i} \bar{\Gamma}_{i}^{R},
$$

where the relations among the anomalous dimensions fields, parameters and $\gamma_{i}$ have the forms:

$$
\gamma_{1}=2 \gamma_{\psi} \quad \gamma_{2}=2 \gamma_{\psi}+\gamma_{D} \quad \gamma_{3}=2 \gamma_{\psi}+\gamma_{D}+\gamma_{\tau} \quad \gamma_{4}=3 \gamma_{\psi}+\gamma_{D}+\gamma_{\lambda} .
$$

Taking into account the renormalization scheme, the anomalous dimension can be presented in terms of renormalized derivatives of the one-particle irreducible Green function $\bar{\Gamma}_{i}$ at the normalization point [6-8]. The renormalization procedure is implemented on the diagram in terms of the $\mathcal{R}$ operation. Let us define functions $f_{i}$ by means of the $\mathcal{R}$ operation

$$
\left.f_{i} \equiv \mathcal{R}\left[-\tilde{\tau} \partial_{\tilde{\tau}} \bar{\Gamma}_{i}(\tilde{\tau})\right]\right|_{\tilde{\tau}=1},
$$

The relation for anomalous dimensions in terms of the quantities $f_{i}$ with respect to $\tilde{\tau}$ in the NP is

$$
\gamma_{i}=\frac{2 f_{i}}{1+f_{2}}, \quad i=1,2,4 \text {. }
$$


The main advantage of these considerations is that the $\mathcal{R}$ operation can be expressed in terms of the subtracting operation $1-\mathcal{K}_{i}[4]$, which eliminates all divergences from the Feynman graphs $\mathcal{R} \Gamma=$ $\Pi\left(1-\mathcal{K}_{i}\right) \Gamma$, where the product runs over all relevant subgraphs of a given Feynman diagram, including the diagram as a whole. Then we can obtain the representation for the $\mathcal{R}$ operation $[6,8]$ in the nullmomentum (NM) scheme

$$
\mathcal{R} \chi=\prod_{i} \frac{1}{n_{i} !} \int_{0}^{1} d a_{i}\left(1-a_{i}\right)^{n_{i}} \partial_{a_{i}}^{n_{i}+1} \chi(\{a\}),
$$

where the product is taken over all one-irreducible subgraphs $\chi_{i}$ (including the diagram $\chi$ as a whole) with canonical dimensions $n_{i} \geq 0$ and $a_{i}$ are parameters that stretch the moments flowing into the $i$-th subgraph inside the given graph.

\section{Contribution of Feynman diagrams}

Calculation of contribution to graphs was easily performed up to the two-loop approximation [10] in the time-momentum representation. In the three-loop, most Feynman diagrams $[1,10]$ are evaluated in the same way. Exceptions are 3 diagrams from $\Gamma_{\tilde{\psi} \psi}$ and 9 diagrams from $\Gamma_{\tilde{\psi} \psi \psi}$ (Fig. 1). All these diagrams have one common feature, they contain two 1-loop subgraphs.

(A)

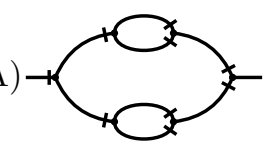

(B)

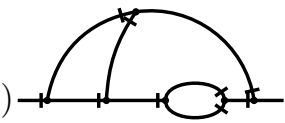

$(\mathrm{E})$

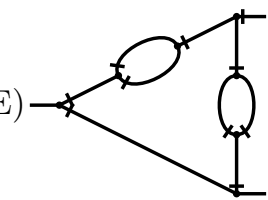

(I)

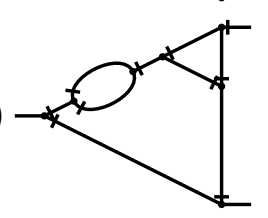

$(\mathrm{F})$

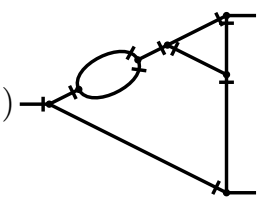

$(\mathrm{J})$

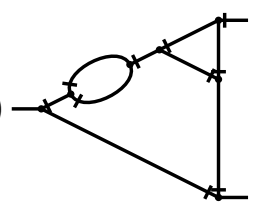

(C)

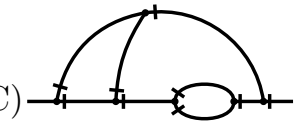

(G)

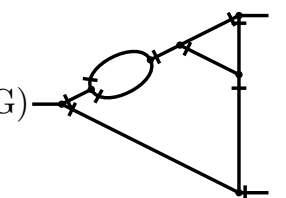

$(\mathrm{K})$

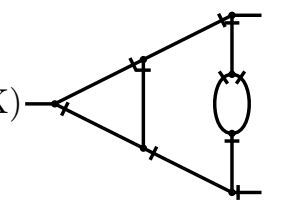

(D)

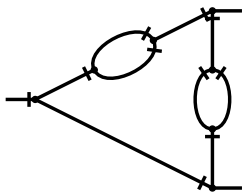

$(\mathrm{H})$

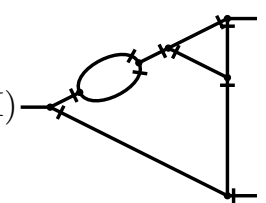

(L)

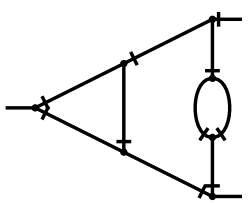

Figure 1. Three-loop Feynman diagrams which can not be calculated in the time-momentum representation. The diagrams (A) - (C) belong to $\Gamma_{\tilde{\psi} \psi}$ and the diagrams (D) - (L) are part of $\Gamma_{\tilde{\psi} \psi \psi}$.

One of the possible ways to solve this problem was to calculate the contribution of the 1-loop diagram $\Gamma_{\tilde{\psi} \psi}$ and obtain prescription when the divergent part of diagram has the form

$$
\begin{aligned}
\text { quadratic: } & P_{R_{2}}=\frac{\Gamma(\varepsilon) \Gamma(2-\varepsilon)}{4} \frac{1}{\varepsilon-1}\left[\left(\frac{-i \omega}{2}+\frac{p^{2}}{4}+1\right)^{1-\varepsilon}-1-(1-\varepsilon)\left(\frac{-i \omega}{2}+\frac{p^{2}}{4}\right)\right], \\
\text { logarithmic: } & \dot{P}_{R_{0}}=\frac{\Gamma(\varepsilon) \Gamma(2-\varepsilon)}{4}\left[\left(\frac{-i \omega}{2}+\frac{p^{2}}{4}+1\right)^{-\varepsilon}-1\right],
\end{aligned}
$$


where $\tau=1, \Gamma(\cdot)$ denotes Gamma functions, $p$ and $\omega$ denote the external momentum and frequency with respect to the subgraph. The diffusion constant $D$ is excluded. In the case of 1-loop subgraph $\Gamma_{\tilde{\psi} \psi \psi}$ part a logarithmic divergence is obtained

$$
T_{R_{0}}=\frac{\Gamma(\varepsilon) \Gamma(2-\varepsilon)}{8}\left[\int_{0}^{1} d a\left(\frac{-i \omega a}{2}+\frac{p^{2} a}{4}+1-\frac{a^{2} p^{2}}{4}\right)^{-\varepsilon}-1\right] .
$$

The final result of Feynman diagrams (Fig. 1) can be obtained using the relation for the 1-loop subgraphs. In the first step of calculation for the diagram, the integrand is expressed in the frequencymomentum representation. In the second step the $\mathcal{R}$ operation from (7) is applied and the final symbolic integrand for numerical evaluation is found. In this integrand the divergent 1-loop contribution is replaced with respect to the previous relations. The last step is the numerical calculation where the integration is taken with respect to two or three parameters.

Now the final result of all diagrams for $\Gamma_{\tilde{\psi} \psi}$ can be derived. Using relations (8) one arrives to the following expressions for anomalous dimension in three-loop approximation

$$
\begin{aligned}
& \gamma_{1}=u\left(-\frac{1}{8}+\frac{\varepsilon}{16}-\frac{\pi^{2} \varepsilon^{2}}{192}\right)+u^{2}(0.026645-0.042537 \varepsilon)-u^{3} 0.0190775 \\
& \gamma_{2}=u\left(-\frac{1}{16}+\frac{\varepsilon}{32}-\frac{\pi^{2} \varepsilon^{2}}{384}\right)+u^{2}(0.010607-0.016903 \varepsilon)-u^{3} 0.006826
\end{aligned}
$$

Our results from the two-loop approximation with the new way of calculation is in good agreement with previous calculation [10] and also agrees very well with analytic calculation [1] in the $\varepsilon$-expansion. The calculation is in progress for 150 Feynman diagrams $\Gamma_{\tilde{\psi} \psi \psi}$ (in order to obtain $\gamma_{3}, \gamma_{4}$ ).

\section{Acknowledgement}

This research was supported by the Ministry of Education and Science of the Russian Federation (Agreement No. 02.a03.21.0008) and the Ministry of Education, Science, Research, and Sport of the Slovak Republic (VEGA Grant No. 1/0345/17).

\section{References}

[1] H. K. Janssen and U. C. Täuber, Ann. Phys. 315, 147-192, (2004)

[2] J. L. Cardy and R. L. Sugar, J. Phys. A 13, L423-L427, (1980)

[3] H. Hinrichsen, Adv. Phys. 49, 815-958 (2000)

[4] A. N. Vasilev, The Field Theoretic Renormalization Group in Critical Behavior Theory and Stochastic Dynamics ([in Russian], PIYaF, St. Petersburg, 1998; English trans., Chapman and Hall/CRC, Boca Raton, Fla, 2004)

[5] J. C. Collins, Renormalization: an introduction to renormalization, the renormalization group and the operator-product expansion (Cambridge university press, 1984)

[6] L. Ts. Adzhemyan, M. V. Kompaniets, Theor. Math. Phys. 169, 1450-1459 (2011)

[7] L. Ts. Adzhemyan, M. V. Kompaniets, S. V. Novikov, V. K. Sazonov, Theor. Math. Phys. 175(3), 719-728 (2013)

[8] L. Ts. Adzhemyan, M. V. Kompaniets, Journal of Physics Conference Series 523, 012049, (2014)

[9] P. C. Martin, E. D. Siggia, H. A. Rose, Physical Review A, 8 (1), 423, (1973)

[10] L. Ts. Adzhemyan, et al., EPJ Web of Conferences. 108 (EDP Sciences, 2016) 